\title{
Diferencias de género en la enfermedad alcohólica
}

\author{
José Juan Ávila Escribano*; David González Parra** \\ * Psiquiatra. Unidad de Alcoholismo. Salamanca \\ **MIR de Psiquiatría. Servicio de Psiquiatría. Hospital Clínico Universitario. Salamanca \\ Enviar correspondencia a: \\ José Juan Ávila Escribano. C/Toro, 84-90, 3 D. 37002-SALAMANCA. Correo electrónico: javilae@chsaludmental.com
}

Recibido: Noviembre de 2006 Aceptado: Julio de 2007

\section{RESUMEN}

Estudios epidemiológicos recientes señalan que el consumo de alcohol en la mujer ha aumentado en los últimos años, lo que nos hace pensar que en un futuro próximo habrá mayor número de mujeres que padezca alcoholismo. Además, su enfermedad presenta características diferenciales cuyo conocimiento es importante para iniciar el tratamiento lo más precozmente posible.

El objetivo del presente estudio consiste en investigar las diferencias clínicas en los trastornos por uso de alcohol según el sexo de los pacientes. Se realizó en una muestra de 370 pacientes, 325 hombres $(87,8 \%)$ y 45 mujeres $(12,2 \%)$, cuya edad media era 42,83 y 44,60 años, respectivamente. Los pacientes fueron valorados mediante la entrevista estructurada Europasi y estudio analítico con perfil hepático y hemograma.

Los resultados más destacados fueron: las mujeres iniciaban el consumo de alcohol significativamente más tarde que los hombres (19,61 y 16,90 años respectivamente; $p<0,008)$, tenían significativamente más edad cuando el patrón de consumo era problemático $(30,93$ y 24,68 años; $p<0,003)$, llevaban menos años de consumo $(13,26$ versus 17,85 años; $p<0,02)$ y consumían menos gramos de alcohol (117,70 y 133,80 gr.; n.s.). En el Europasi las mujeres obtenían significativamente más puntuación que los hombres en la escala psiquiátrica $(2,91$ y $1,97$ respectivamente; $p<0,007)$ y éstos presentaban más problemas legales que aquellas $(1,20$ y 1,$0 ; p<0,000)$. En las pruebas biológicas, los valores del enzima GGT eran más elevados en los hombres $(137,51)$ que en las mujeres $(96,7)$ aunque esta diferencia no era significativa y el valor del VCM era significativamente mayor en las mujeres $(98,1$ y 95,$05 ; p<0,03)$. Otro hallazgo relevante fue que el porcentaje de mujeres que había acudido a dispositivos privados era mayor que el de hombres (15\% versus $4,6 \% ; p<0,01)$.

Palabras clave: dependencia, alcohol, alcoholismo, diferencias, género, mujeres alcohólicas.

\section{ABSTRACT}

Recent epidemiological studies indicate that alcohol consumption in women has increased in the last few years, which suggests that alcoholism in women will also increase in the near future. Moreover, this disease shows differential characteristics in women, and knowledge of these characteristics is important so that treatment can begin as early as possible.

The objective of the present study was to explore clinical differences in alcohol use disorders according to patients' gender. It was carried out with a sample of 370 patients, 325 men (87.8\%) and 45 women (12.2\%), with mean ages of 42.83 and 44.6 years, respectively. The patients were assessed through the Europasi interview and analytical studies with liver enzyme profiles and blood tests.

The most notable results were: women began alcohol consumption significantly later than men (19.61 and 16.9 years, respectively; $p<0.008)$; they were significantly older than men when the consumption pattern became problematic (30.93 and 24.68 years, respectively; $p<0.003)$; they had been drinking for fewer years (13.26 versus 17.85 years; $p<0.02$ ); and they drank fewer grams of alcohol (117.7 and 133.8 g., respectively; n.s.). Women scored significantly higher than men on the Europasi psychiatric scale (2.91 and 1.97, respectively; $p<0.007$ ) and men had more legal problems than women (1.2 and 1.0, respectively; $\mathrm{p}<0.000$ ). In the biological tests the GGT enzyme values were higher in men (137.51) than in women (96.7), but this difference was not significant, and the VCM value was significantly higher for women (98.1) than for men (95.05). Another important finding was that the percentage of women who had sought private professional help was higher than that of men $(15 \%$ versus $4.6 \% ; p<0.01$ ).

Key words: dependence, alcohol, alcoholism, differences, gender, alcoholic women. 


\section{INTRODUCCIÓN}

a prevalencia global de los trastornos por uso de alcohol (T.U.A.), en los últimos doce meses, en la población general es del 8,46\%, según los estudios epidemiológicos más recientes ${ }^{1}$. Estos estudios ponen de manifiesto que dicha prevalencia es significativamente mayor en los hombres que en las mujeres $\left(12,35 \%\right.$ y $4,87 \%$, respectivamente) ${ }^{1}$ (Tabla 1). Cuando se analizan los datos epidemiológicos de forma más pormenorizada, teniendo en cuenta el diagnóstico (abuso o dependencia de alcohol), edad y raza, se pueden establecer algunas conclusiones: 1) la prevalencia de abuso y dependencia de alcohol en las mujeres es muy similar $(2,55 \%$ y $2,32 \%$, respectivamente) (Tabla 1). 2) Las tasas de abuso y dependencia de alcohol son significativamente mayores en las mujeres de raza caucásica que en los otros grupos raciales. 3) La prevalencia de los T.U.A guarda una relación inversa con la edad, de manera que disminuye a medida que la edad de la población estudiada aumenta, siendo el grupo de edad comprendido entre los 18-29 años el que presenta prevalencias más altas, tanto los hombres como las mujeres ${ }^{1}$ (Figura 1).

Cuando se comparan estos datos con los obtenidos en un estudio similar, realizado en $1991^{2}$, se comprueba que en esta última década ha habido, en primer lugar, un incremento de los T.U.A desde el 7,41 en 1991 hasta el 8,46 en 2001 (Tabla 1). En segundo lugar, este incremento acontece tanto en hombres como en mujeres, pero la proporción de los T.U.A, entre ambos, desciende desde 2,89 en 1991 hasta el 2,55 en el año 2001, produciéndose una discreta con- vergencia. No obstante, hay el doble de hombres con problemas de alcohol que de mujeres.

En nuestro país no poseemos estudios que muestren la prevalencia del abuso y dependencia de alcohol, pero existen estudios epidemiológicos, realizados periódicamente, sobre los hábitos del consumo de alcohol en la población general ${ }^{3}$ (Tabla 2). En estos estudios se constata que el consumo diario de alcohol, tanto en hombres como en mujeres se ha mantenido estable en los últimos años y los porcentajes de personas que consumen alcohol en cantidades que suponen un riesgo para su salud física y/o psíquica, tampoco han experimentación variación alguna. Finalmente, la edad media de inicio del consumo de alcohol es prácticamente la misma (16,7-16,9 años) en la última década.

A pesar de los datos expuestos, que nos inclinan a pensar que los T.U.A. en la mujer pueden estar incrementando o lo harán en el futuro, la proporción de mujeres que acude a tratamiento por estos trastornos es relativamente bajo. Algunos estudios sobre el alcoholismo femenino han señalado que la proporción hombres/mujeres es de 4/1 o inferior ${ }^{4,5}$.

Por otra parte, en la literatura científica se ha señalado, muy frecuentemente, que la dependencia de alcohol en el sexo femenino presenta una serie de características etiológicas y clínicas que le diferencian del alcoholismo en el hombre 4, 6, 7, 8, 9, 10, 11, 12, 13 .

Se ha descrito que las mujeres presentan, específicamente, una serie de factores etiológicos adicionales que aumentan el riesgo de padecer alcoholismo. Los más sobresalientes son: un patrón de consumo

Tabla 1. Prevalencia anual de los Trastornos por Uso de Alcohol ${ }^{(1,2)}$

\begin{tabular}{|l|c|c|c|}
\hline & HOMBRES (\%) & MUJERES (\%) & TOTAL (\%) \\
\hline Abuso de Alcohol (1991) & 4.67 & 1.51 & 3.03 \\
\hline Dependencia de Alcohol (1991) & 6.33 & 2.58 & 4.38 \\
\hline Prevalencia global de T.U.A. (1991) & 11.00 & 4.09 & 7.41 \\
\hline Abuso de Alcohol (2001) & 6.93 & 2.55 & 4.65 \\
\hline Dependencia de Alcohol (2001) & 5.42 & 2.32 & 3.81 \\
\hline Prevalencia global T.U.A. (2001) & 12.35 & 4.87 & 8.46 \\
\hline
\end{tabular}

Tabla 2. Consumo de alcohol en la población española ${ }^{(3)}$

\begin{tabular}{|c|c|c|c|c|c|c|c|c|}
\hline \multirow{2}{*}{$\begin{array}{l}\text { AÑO } \\
\text { Género }\end{array}$} & \multicolumn{2}{|c|}{1997} & \multicolumn{2}{|c|}{1999} & \multicolumn{2}{|c|}{2001} & \multicolumn{2}{|c|}{2003} \\
\hline & Hombre & Mujer & Hombre & Mujer & Hombre & Mujer & Hombre & Mujer \\
\hline Consumo anual & 86.4 & 70.5 & 83.2 & 67.2 & 85.2 & 70.9 & 84.5 & 68.6 \\
\hline Consumo mensual & 75.2 & 52.1 & 74.4 & 49.1 & 76.4 & 50.9 & 75.8 & 52.1 \\
\hline Consumo diario & 20.2 & 5.2 & 21.4 & 6.1 & 24.5 & 6.8 & 21.9 & 6.2 \\
\hline Consumo de riesgo & 8 & 3.4 & 5.2 & 2.5 & 7.6 & 3.5 & 7.4 & 3.1 \\
\hline Edad media inicio & \multicolumn{2}{|c|}{16.8} & \multicolumn{2}{|c|}{16.9} & \multicolumn{2}{|c|}{16.9} & \multicolumn{2}{|c|}{16.7} \\
\hline
\end{tabular}




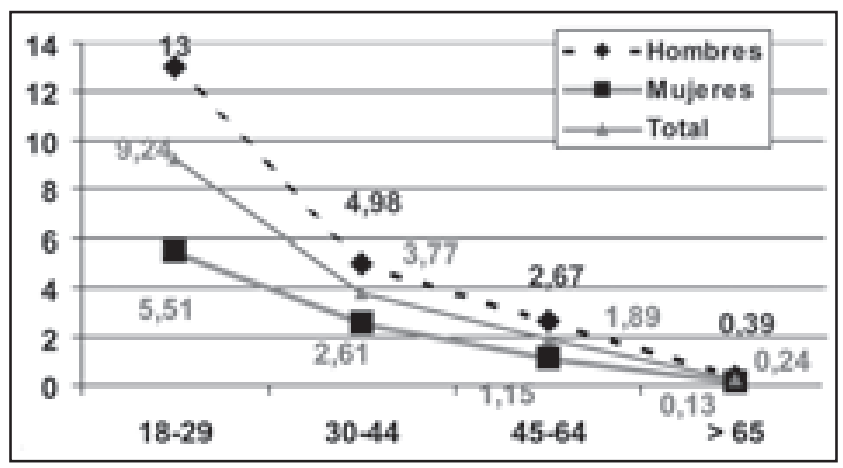

Figura 1. Prevalencia de la dependencia de alcohol según edad y género ${ }^{(1,2)}$

excesivo de alcohol por parte de su pareja, la presencia de síntomas depresivos y/o depresión franca que además influye en la cronificación de la dependencia alcohólica, antecedentes de abuso sexual en la infancia y malos tratos en la niñez o adultez ${ }^{9}, 11,13$.

Las diferencias clínicas que se resaltan en dichos estudios, expuestas de forma esquemática son: 1) Las mujeres inician el consumo de alcohol a una edad más tardía, consumen menos cantidad y acuden a consulta a una edad similar o menor que los varones, por lo que la enfermedad se desarrolla en un corto periodo de tiempo ${ }^{6}$. 2) La presencia de síntomas psicopatológicos, principalmente depresivos, es muy destacada, como anteriormente se mencionaba, siendo éstos el motivo principal de consulta. En relación con este punto, los intentos de autolisis son muy frecuentes en la historia psicopatológica de estas mujeres ${ }^{7,12}$. 3) El consumo de otras drogas, principalmente psicofármacos y estimulantes, asociado al consumo de alcohol, es un hallazgo muy frecuente. 4) Las consecuencias somáticas y familiares derivadas del uso de alcohol tienen más incidencia en el alcoholismo femenino que en el masculino; mientras que los problemas legales, laborales y sociales lo son menos ${ }^{6}$. Se ha planteado como hipótesis que el alcohol es más perjudicial en la mujer que en el hombre a dosis equivalentes, bien por mecanismos farmacocinéticos que originan concentraciones más elevadas de alcohol en sangre, bien por mayor sensibilidad del sistema nervioso al alcohol ${ }^{13,14}$.

En nuestro medio existen pocos trabajos que hayan estudiando las características del alcoholismo femenino. Algunos se limitan a realizar análisis descriptivos de muestras de mujeres alcohólicas ${ }^{10,15} \mathrm{O}$ son revisiones sobre este tema ${ }^{13}$. El objetivo del presente trabajo era investigar si existían diferencias clínicas de los trastornos por uso de alcohol según el sexo de los pacientes en cuanto a rapidez en el desarrollo de la adicción y severidad de las complicaciones de la misma.

\section{MÉTODOS}

El presente trabajo se realizó en la Unidad de Alcoholismo de Salamanca. Se trata de un estudio comparativo, según el sexo, de las características de la enfermedad alcohólica que presentaba una muestra de pacientes alcohólicos que fueron atendidos en dicha Unidad desde Junio del 2002 hasta Diciembre del 2004, ambos inclusive. Durante ese periodo se atendieron a 413 paciente de los cuales 361 (87,4\%) eran hombres y $52(12,4 \%)$ mujeres.

Todos los pacientes fueron valorados, antes de iniciar tratamiento, mediante la entrevista estructurada Europasi ${ }^{16}$ que es una adaptación española del Índice de Severidad de la Adicción (ASI) y valora la repercusión del consumo de alcohol en diferentes áreas: somática, psiquiátrica, adictiva, laboral, legal y familiar. También se recogieron de forma sistemática algunas manifestaciones del alcoholismo que son importantes y no están recogidas en dicha entrevista como presencia de síntomas de abstinencia, polineuropatía, palimpsestos, convulsiones y agresividad, que fueron valorados clínicamente. Así mismo se calcularon los gramos de alcohol ingerido diariamente. Además se les realizó estudio analítico con perfil hepático y hemograma completo con el objetivo de valorar la repercusión somática de la enfermedad en el momento actual y su evolución a lo largo del seguimiento.

El diagnóstico de los pacientes se realizó siguiendo los criterios del DSM-IV de la Asociación Psiquiátrica Americana ${ }^{17}$. Se recogía como diagnóstico principal el tipo de trastorno por uso de alcohol (dependencia o abuso de alcohol) y como diagnóstico secundario el trastorno mental que existiese concomitantemente en el momento de iniciar tratamiento. Entre los diagnósticos secundarios se incluían trastornos de personalidad, del estado de ánimo, abuso y/o dependencia de cocaína, juego patológico, trastornos de ansiedad, trastornos psicóticos y trastornos del desarrollo intelectual.

Como uno de los objetivos del estudio era la evaluación y comparación de la repercusión somática del alcohol en ambos sexos, se excluyeron los pacientes que padecían hepatitis $\mathrm{C}$ de tipo crónico. Así pues el tamaño muestral del estudio fue de 370 pacientes, 325 hombres $(87,8 \%)$ y 45 mujeres $(12,2 \%)$, cuya edad estaba comprendida entre los 19 y 76 años.

El tratamiento estadístico se realizó mediante el programa informático SSPS, versión 10.0. Se determinaron la frecuencia de las variables cualitativas y la media con desviación típica de las variables cuantitativas. Los estadísticos que se utilizaron para determinar el grado de significación fueron la " $t$ " de Student y el $\mathrm{Chi}^{2}$. 


\section{RESULTADOS}

\section{Características sociodemográficas}

Las características sociodemográficas de la muestra, según el sexo, están recogidas en la Tabla 3. El $87,8 \%$ de la población estudiada era del sexo masculino y el $12,2 \%$ del sexo femenino con lo que la proporción de hombres y mujeres en nuestro estudio era de 7/1. La edad media de las mujeres que acudieron a consulta (44,60 años) era mayor que la de los hombres $(42,83$ años), pero esta diferencia no era significativa. Prácticamente la mitad de las mujeres (49\%) procedía del medio rural, mientras que más de la mitad de los hombres $(63,4 \%)$ vivía en la ciudad, pero esta diferencia tampoco era significativa. En cuanto al estado civil, ambos grupos eran muy similares: más de la mitad estaban casados ( $57,1 \%$ versus $58,1 \%$ ), el porcentaje de hombres separados $(18,5 \%)$ era significativamente superior al de mujeres $(9,3 \%)$ y en las mujeres el porcentaje de viudez $(11,6 \%)$ era significativamente mayor que en los hombres $(0,6 \%)$. El nivel de estudios alcanzado por la muestra era bajo pues más de dos terceras partes poseía estudios primarios o graduado escolar $(76,8 \%$ los hombres y $66,6 \%$ las mujeres).
En cuanto al tipo de empleo de la muestra aparecían dos hallazgos que diferían significativamente en ambos grupos. El $71,8 \%$ de los hombres estaba empleado, mientras que las mujeres solo el $28,2 \%$ tenía empleo y el 33,3\% se dedicaba a las labores de la casa exclusivamente.

\section{Características clínicas}

Entre las características clínicas del alcoholismo hay que incluir cuatro apartados: historia del consumo de alcohol y grado del mismo, las repercusiones de la enfermedad en los diferentes campos recogidos en el Europasi y la presencia de otras manifestaciones características de la enfermedad que no vienen recogidas en el citado cuestionario, las determinaciones de las pruebas de laboratorio practicadas y la existencia y tipo de tratamiento del alcoholismo antes de acudir a la Unidad.

Las mujeres iniciaban el consumo de alcohol más tarde que los hombres $(19,61$ y 16,90 años respectivamente), diferencia de edad que era significativa (Tabla 4). También éstas tenían significativamente más edad que los hombres cuando el patrón de consumo de alcohol se volvía regular y problemático $(30,93$ y 24,68 años respectivamente) (Tabla 4). La duración o

Tabla 3. Características sociodemográficas, según sexo

\begin{tabular}{|c|c|c|c|}
\hline & HOMBRES (N=325) \% & MUJERES (N=45) \% & Significación \\
\hline EDAD MEDIA & $42.83 \pm 11.9$ & $44.60 \pm 12.04$ & 0.35 \\
\hline \multicolumn{4}{|l|}{ ESTADO CIVIL } \\
\hline Casado & 57.1 & 58.1 & \\
\hline Soltero & 23.8 & 20.9 & \\
\hline Separado & 18.5 & 9.3 & $0.001 *$ \\
\hline Viudo & 0.6 & 11.6 & $0.001^{*}$ \\
\hline RESIDENCIA & & & 0.17 \\
\hline Gran ciudad & 63.4 & 51.1 & \\
\hline Pequeña ciudad & 5.5 & 11.1 & \\
\hline Rural & 31.1 & 37.8 & \\
\hline ESTUDIOS & & & 0.36 \\
\hline Primarios & 50.2 & 41.0 & \\
\hline Graduado & 26.6 & 25.6 & \\
\hline Bachillerato/FP & 23.3 & 33.3 & \\
\hline \multicolumn{4}{|l|}{ EMPLEO } \\
\hline Tiempo completo & 71.8 & 28.2 & $0.000^{*}$ \\
\hline Tiempo parcial & 6.4 & 17.9 & \\
\hline Paro & 7.0 & 10.3 & \\
\hline Estudiante & 1.0 & 0 & \\
\hline Militar & 0.7 & 0 & \\
\hline Jubilado & 11.7 & 10.3 & \\
\hline Ama de casa & 0 & 33.3 & $0.000^{*}$ \\
\hline
\end{tabular}


Tabla 4.- Historia de consumo de alcohol

\begin{tabular}{|l|c|c|c|c|c|}
\hline & SEXO & N & Media & D. T. & Significación \\
\hline \multirow{2}{*}{ Inicio alcohol } & Hombre & 304 & 16.90 & 4.36 & $0.008^{* *}$ \\
\hline \multirow{2}{*}{ Consumo regular } & Mujer & 41 & 19.61 & 6.09 & \\
\hline \multirow{2}{*}{ Duración consumo } & Hombre & 305 & 24.68 & 9.01 & $0.003^{* *}$ \\
\cline { 2 - 6 } & Mujer & 43 & 30.93 & 12.47 & \\
\hline \multirow{2}{*}{ Gramos alcohol } & Hombre & 305 & 17.85 & 12.44 & $0.02^{*}$ \\
\cline { 2 - 7 } & Mujer & 43 & 13.26 & 10.46 & \\
\hline
\end{tabular}

Tabla 5.- Resultados EUROPASI

\begin{tabular}{|c|c|c|c|c|c|}
\hline & SEXO & $\mathrm{N}$ & Media & D.T. & Significación \\
\hline \multirow{2}{*}{ Severidad problemas médicos } & Hombre & 325 & 1.48 & 1.29 & 0.4 \\
\hline & Mujer & 45 & 1.33 & .95 & \\
\hline \multirow{2}{*}{ Severidad problemas psiquiátricos } & Hombre & 325 & 1.97 & 1.81 & $0.007^{* *}$ \\
\hline & Mujer & 45 & 2.91 & 2.16 & \\
\hline \multirow{2}{*}{ Severidad problemas con alcohol } & Hombre & 299 & 6.99 & 1.30 & 0.2 \\
\hline & Mujer & 40 & 7.25 & 1.46 & \\
\hline \multirow{2}{*}{ Severidad problemas con drogas } & Hombre & 295 & .69 & 2.00 & 0.4 \\
\hline & Mujer & 40 & .43 & 1.45 & \\
\hline \multirow{2}{*}{ Severidad problemas laborales } & Hombre & 298 & 2.49 & 2.56 & 0.3 \\
\hline & Mujer & 37 & 2.89 & 2.86 & \\
\hline \multirow{2}{*}{ Severidad problemas legales } & Hombre & 296 & 1.20 & .85 & $0.000 * *$ \\
\hline & Mujer & 38 & 1.00 & .00 & \\
\hline \multirow{2}{*}{ Severidad problemas familiares } & Hombre & 185 & 4.28 & 2.43 & 0.9 \\
\hline & Mujer & 27 & 4.26 & 2.73 & \\
\hline
\end{tabular}

Tabla 6.- Otras manifestaciones clínicas de los trastornos por uso de alcohol.

\begin{tabular}{|l|c|c|c|c|c|}
\hline \multirow{2}{*}{} & \multicolumn{2}{|c|}{ HOMBRES } & \multicolumn{2}{c|}{ MUJERES } & Significación \\
\cline { 2 - 6 } & N & $\%$ & N & $\%$ & \\
\hline Síntomas de abstinencia & 195 & 60.2 & 27 & 60.0 & 0.5 \\
\hline Síntomas de polineuropatía & 175 & 54.0 & 25 & 55.6 & 0.5 \\
\hline Palimpsestos & 210 & 65.0 & 29 & 64.4 & 0.5 \\
\hline Convulsiones & 20 & 6.2 & 1 & 2.3 & 0.2 \\
\hline
\end{tabular}

años de consumo, como consecuencia de lo mencionado anteriormente, era significativamente menor en las mujeres que en los hombres $(13,26$ versus 17,85 años) (Tabla 4). Sin embargo, los hombres consumían más cantidad de alcohol (133,88 gr./día), aunque esta diferencia no era significativa.

En cuanto a la magnitud y severidad de las consecuencias ocasionadas por el alcohol en las diferentes áreas valoradas por el cuestionario Europasi, se pudo apreciar que las puntuaciones eran similares en ambos grupos de la muestra, como queda recogido en la Tabla 5. No obstante, se observó que existían diferencias significativas en dos áreas. Las mujeres presentaban más problemas psicopatológicos que los hombres (2,91 y 1,97 respectivamente) y éstos presentaban más problemas legales que aquellas $(1,20$ y 1,0 respectivamente).

Mas de la mitad de los pacientes de ambos grupos refería síntomas de abstinencia del alcohol (60\%), manifestaciones de polineuropatía (54\%) y palimpsestos (65\%), sin existir diferencias entre los sexos (Tabla 6). 
Tabla 7.- Resultados analíticos

\begin{tabular}{|c|c|c|c|c|c|}
\hline & SEXO & N & Media & D.T. & Significación \\
\hline \multirow{2}{*}{ GOT } & Hombre & 242 & 45.77 & 44.88 & 0.8 \\
\cline { 2 - 6 } & Mujer & 35 & 47.69 & 58.36 & 39.62 \\
\multirow{2}{*}{ GPT } & Hombre & 242 & 46.31 & 47.89 & 0.5 \\
\cline { 2 - 6 } & Mujer & 35 & 41.29 & 266.69 & 0.3 \\
\hline \multirow{2}{*}{ GGT } & Hombre & 227 & 137.51 & 148.38 & \\
\cline { 2 - 6 } & Mujer & 35 & 96.74 & 6.894 & $0.03^{*}$ \\
\hline \multirow{2}{*}{ VCM } & Hombre & 236 & 95.395 & 7.573 & \\
\hline
\end{tabular}

Tabla 8.-Tratamientos previos de la dependencia de alcohol y tratamientos psiquiátricos

\begin{tabular}{|l|c|c|c|c|c|}
\hline & \multicolumn{2}{|c|}{ HOMBRES } & \multicolumn{2}{c|}{ MUJERES } & Significación \\
\hline & $\mathbf{N}$ & $\%$ & $\mathbf{N}$ & $\%$ & \\
\hline Tratamiento ambulatorio & 104 & 34.3 & 11 & 27.5 & 0.2 \\
\hline Ingresos Servicio Psiquiatría desintoxicación & 19 & 6.3 & 5 & 12.5 & 0.1 \\
\hline Otro tipo de tratamiento (Comunidad Terapéutica) & 37 & 12.2 & 3 & 7.5 & 0.2 \\
\hline Tratamiento servicio privado & 14 & 4.6 & 6 & 15 & $0.01^{*}$ \\
\hline Tratamiento psiquiátrico ambulatorio & 86 & 26.5 & 16 & 35.6 & 0.1 \\
\hline Ingreso psiquiátrico & 27 & 7.7 & 4 & 8.9 & 0.4 \\
\hline
\end{tabular}

En la Tabla 7 se exponen los resultados más significativos de las pruebas analíticas practicadas que expresan la repercusión hepática y hematológica del alcohol y son pruebas coadyuvantes para el diagnóstico de la enfermedad. Se encontró que los valores del enzima GGT eran más elevados en los hombres $(137,51)$ que en las mujeres $(96,7)$ aunque esta diferencia no era significativa. Por el contrario, el valor del VCM era significativamente mayor en las mujeres $(98,1)$ que en los hombres $(95,05)$.

Finalmente, otro aspecto que indica la problemática que el consumo excesivo de alcohol acarrea es la presencia de tratamientos previos para este problema y el tipo de tratamiento recibido. El 34,3\% de varones ya había recibido tratamiento ambulatorio en alguna ocasión, mientras que el porcentaje de mujeres era el $27,5 \%$. Además, 19 hombres $(6,3 \%)$ y 5 mujeres $(12,5 \%)$ habían estado ingresados en el Servicio de Psiquiatría anteriormente para tratamiento del síndrome de abstinencia; aunque el porcentaje de mujeres era superior, la diferencia no era significativa. También 37 hombres $(12,2 \%)$ y 3 mujeres $(7,5 \%)$ habían estado en otros dispositivos asistenciales del servicio público como Comunidades terapéuticas. Hay que destacar que el porcentaje de mujeres (15\%) que había acudido previamente a dispositivos privados era significativamente mayor que el de hombres (4,6\%) (Tabla 8).

El cuestionario Europasi recoge, también, si los pacientes habían estado en tratamiento psiquiátrico ambulatorio y/o habían estado ingresados por trastor- nos psiquiátricos. En la muestra del presente trabajo 86 hombres $(26,5 \%)$ y 16 mujeres $(35,6 \%)$ habían recibido tratamiento por los equipos de salud mental a causa de diferentes trastornos psiquiátricos, diferencia que no era significativa. Tampoco había diferencias entre ambos grupos en cuanto a la existencia de ingresos psiquiátricos (Tabla 8).

\section{Características diagnósticas}

El 77,8\% de mujeres del estudio fue diagnosticado de dependencia de alcohol y el 22,2\% de abuso de alcohol. Estos resultados diagnósticos fueron muy similares a los de la muestra de hombres: el 71,1\% fue diagnosticado de dependencia de alcohol y el $28,9 \%$ de abuso.

Además de este diagnóstico principal que era el que motivaba la solicitud de ayuda terapéutica, 126 pacientes $(34,1 \%)$ fueron diagnosticados de otro trastorno psicopatológico asociado al trastorno por uso de alcohol, de los cuales 111 (88,1 \%) eran varones y 15 $(11,8 \%)$ mujeres. En el total de la muestra, los diagnósticos más frecuentes fueron trastorno de personalidad $(11,9 \%)$, trastorno depresivo $(8,1 \%)$ y abuso/dependencia de cocaína $(9,5 \%)$. Cuando se analizaron estos diagnósticos según el sexo se pudo comprobar que los diagnósticos más frecuentes, en los hombres, eran el trastorno de personalidad y el abuso/dependencia de cocaína, mientras que en las mujeres el más frecuente era el trastorno depresivo (Tabla 9). 
Tabla 9.- Diagnósticos asociados al T.U.A. en la muestra total

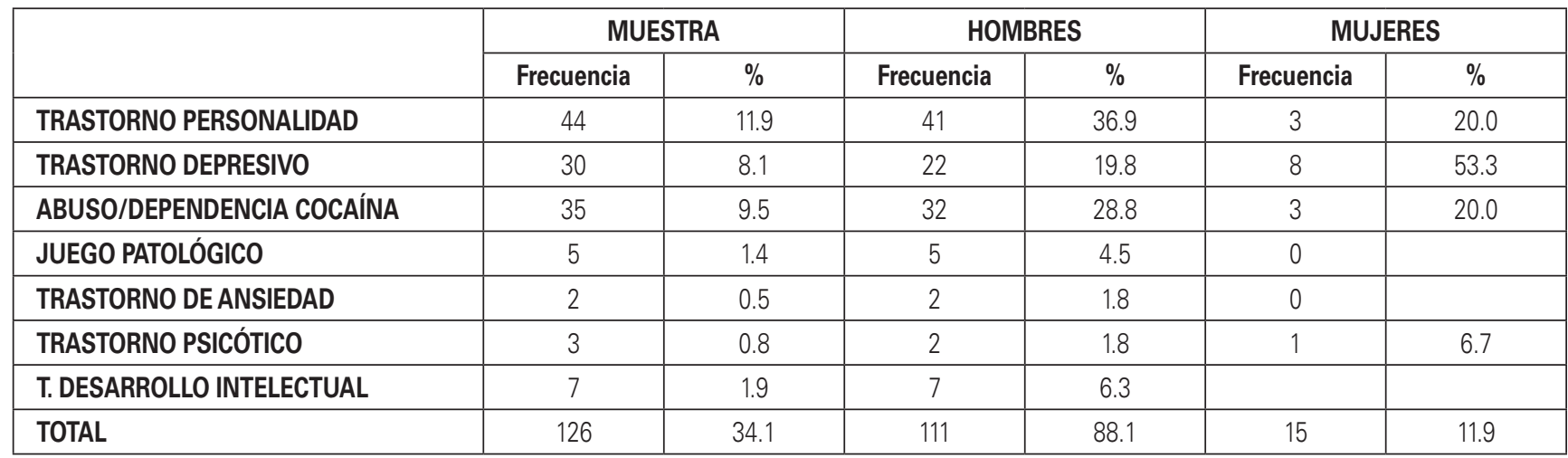

\section{DISCUSIÓN}

El perfil de la mujer con problemas de alcohol del presente estudio se caracterizaba por ser una persona en la cuarta década de la vida (44,60 años), generalmente casada, con estudios primarios y ama de casa. El perfil de los hombres atendidos en la Unidad de Alcoholismo presentaba características sociodemográficas parecidas (varón de 42,83 años, casado, con estudios primarios, residente en la ciudad y con trabajo remunerado). Cuando se compara el perfil de la mujer, descrito en este estudio, con el que aparece en otros trabajos realizados en nuestro medio, se comprueba que las características son similares ${ }^{10,15}$. Más concretamente, Avila et al. ${ }^{10}$ realizaron un estudio descriptivo de mujeres con alcoholismo que fueron atendidas en la década precedente (entre 1985 y 1995) y el perfil que aparecía en ese estudio era prácticamente idéntico al del presente trabajo. Sin embargo en el estudio de Morales et al..$^{15}$ se aprecia que la edad media de su muestra, cuando inicia tratamiento, era menor (37 años).

Hay un hallazgo en nuestro estudio que llama poderosamente la atención y es la baja proporción de mujeres que acuden a tratamiento en comparación con los hombres. En nuestra muestra, la proporción era de $1 / 7$ y en el estudio de Morales et al..$^{15}$, anteriormente citado era de $1 / 6$. Sin embargo, los estudios epidemiológicos que se han realizado sobre la prevalencia de los T.U.A. señalan que la proporción, según el sexo, es el 2,5, es decir, aproximadamente cada 3 hombres hay una mujer con alcoholismo'. En nuestro país, la proporción de consumidores de alto riesgo, entre hombres y mujeres, es el $2,4^{3}$. Ante este hecho surgen algunos interrogantes: ¿las mujeres acuden menos a tratamiento o acuden menos a los dispositivos terapéuticos convencionales?, ¿cuáles serían las razones para explicar este fenómeno?, ¿ los trastornos por uso de alcohol son más difíciles de detectar, por el sistema sanitario, en este grupo de población?, ¿el diagnóstico de abuso y dependencia de alcohol recibe o merece menos atención cuando existen otros síntomas psicopatológicos?.

En cuanto a la primera cuestión, algunos estudios han mencionado la connotación socialmente negativa que tiene esta enfermedad que es más acusada si es mujer quien la padece ${ }^{4,12,18}$. También puede influir el hecho de que la enfermedad pase más desapercibida en el entorno en el que la mujer se desenvuelve como en el ámbito laboral y, principalmente, en el seno familiar, como sucede en nuestro estudio en el que una tercera parte de las mujeres eran amas de casa. Por otra parte, algunos estudios destacan que las mujeres consumen alcohol de forma solitaria, contribuyendo a una menor percepción de este problema ${ }^{12,}$ ${ }^{18}$. Finalmente, el tercer motivo que explique por qué el número de mujeres que han acudido a tratamiento es menor del esperado, puede ser debido a que éstas buscan otros recursos terapéuticos, alejados o no integrados en el sistema público de salud. Este argumento parece estar corroborado por un hallazgo de nuestro estudio, consistente en que el $16 \%$ de las mujeres había estado en tratamiento en otros dispositivos, previamente.

En segundo lugar, es posible que exista una mayor dificultad diagnóstica de estos trastornos en las mujeres. Aunque los cuestionarios como CAGE, AUDIT y TWEAK son muy eficaces en la detección del alcoholismo en la población general, algunos autores ${ }^{19}$ han señalado que cuando se aplican a la población femenina aparece un porcentaje importante de falsos negativos por lo que recomiendan un descenso de la puntuación de corte.

Finalmente, las mujeres con diagnóstico de abuso o dependencia del alcohol presentan comorbilidad psiquiátrica muy frecuentemente, destacando los trastornos depresivos, de ansiedad e insomnio, entre otros ${ }^{9}$, 20. Probablemente muchas mujeres sean diagnosticadas y tratadas del trastorno comórbido, pasando desapercibidos los trastornos por uso de alcohol, en cuyo 
caso si no se diagnostican aparecen como menos frecuentes desde el punto de vista epidemiológico.

Nuestro estudio ha confirmado los resultados que otros autores han descrito acerca de la trayectoria del consumo de alcohol en las mujeres y que han definido como "telescoping course"12. Es decir, historia de consumo más corta, pues entran en contacto con el alcohol a una edad más tardía, y con una afectación somática y sociofamiliar parecida o más severa que los hombres, a pesar de consumir cantidades inferiores de alcohol.

En muchos trabajos se menciona que las mujeres alcohólicas presentan patología hepática más severa que los hombres, así como mayor afectación cardiovascular, miopatía y osteoporosis, consumiendo menores cantidades de alcohol ${ }^{12,13,21}$. Se han propuesto algunas hipótesis para explicar esta mayor morbilidad del alcohol en el sexo femenino, aunque todas hacen hincapié en las diferencias farmacocinéticas que existen entre ambos géneros ${ }^{22,23}$. Las mujeres, cuando consumen dosis de alcohol equivalentes a las de los hombres, consiguen niveles de alcoholemia superiores y presentan una metabolización más rápida del mismo, lo que puede generar mayores cantidades de acetaldehído, producto muy tóxico para el organismo ${ }^{22,23}$. En nuestra muestra de mujeres el nivel de consumo era muy importante, aunque menor que en el grupo masculino pero sin ser significativo, sin embargo la afectación hepática, valorada mediante las determinaciones enzimáticas, era similar a la que presentaban los pacientes alcohólicos. Además, tampoco existían diferencias, entre ambos sexos, en la puntuación de la escala del Europasi que mide la severidad de la patología somática. Con los datos obtenidos en nuestro estudio podemos afirmar que la afectación somática en el grupo de mujeres con alcoholismo es muy similar a la que presenta el grupo de hombres, siendo la cantidad de alcohol ingerida en ambas muestras muy parecida.

Hay datos que ponen de manifiesto una relación directa entre cantidad consumida de alcohol y el cáncer de mama ${ }^{12,21}$. Recientemente, Jiang et al. ${ }^{24}$, en un estudio en el que valoraban la dosis ingerida de alcohol y los niveles de ácido fólico en una muestra de mujeres seguidas prospectivamente durante 16 años, comprobaron que existía una relación directa entre consumo excesivo de alcohol y bajos niveles de ácido fólico con la aparición de cánceres de mama y colon. Estos mismos autores proponían aportar ácido fólico en la dieta como medida preventiva de este tipo de patologías. Un hallazgo que hemos encontrado en nuestro estudio es la elevación del volumen corpuscular medio (VCM), significativamente mayor en las mujeres que en los hombres $(98,1$ y 95,05, respectivamente), aunque en la valoración analítica de nuestra muestra no se determinaban los niveles de ácido fólico, ni vitamina $B_{12}$, responsables ambas del VCM elevado en pacientes con dependencia de alcohol. ¿Existe una mayor alteración del metabolismo y transporte del ácido fólico en las mujeres?. Si la respuesta es afirmativa, explicaría el hallazgo de nuestro estudio y sería conveniente añadir ácido fólico al tratamiento de las mujeres con problemas de alcohol.

La presencia de otros trastornos psiquiátricos en el curso de la dependencia de alcohol es muy frecuente, como se ha mencionado anteriormente, y plantea serios dilemas etiológicos, diagnósticos y terapéuticos 25,26 . En el estudio NCS (National Comorbity Survey) ${ }^{27}$ casi un tercio $(29,2 \%)$ de los pacientes que fueron diagnosticados de dependencia de alcohol en el año previo, habían tenido un trastorno afectivo (odds ratio $=3,6$ ). Como señala Shivani ${ }^{26}$, las manifestaciones psicopatológicas que aparecen en el contexto de un T.U.A. pueden deberse a tres causas: manifestaciones psicopatológicas relacionadas con la enfermedad alcohólica, trastornos psiquiátricos inducidos por el alcohol, trastornos que concurren en un mismo periodo de tiempo pero independientes. En relación a la primera causa, las manifestaciones psicopatológicas que presentan los pacientes, son ocasionadas por el efecto directo del alcohol en el cerebro y por las consecuencias interpersonales y sociolaborales secundarias al consumo de alcohol ${ }^{26}$. Además son más frecuentes en las mujeres que en los hombres y constituyen el motivo principal que induce a solicitar ayuda12, 26 . En nuestro estudio, un tercio de la muestra $(34,1 \%)$ presentaba un trastorno comórbido, no existiendo diferencias entre hombres y mujeres (34\% y 33,3\%, respectivamente). Llama la atención que el 50\% de éstas presentaba un trastorno depresivo que coincide con lo que describen los trabajos anteriormente citados $^{12,25,26}$. Sin embargo, y en ligera contraposición con lo mencionado anteriormente, las mujeres presentaban significativamente mayor puntuación en la escala psiquiátrica que los hombres, aunque esta escala recoge la presencia de síntomas psicopatológicos en el momento de la entrevista y en el mes previo; es decir, hace una valoración de las manifestaciones psicopatológicas que están directamente relacionadas con el T.U.A., según la clasificación de Shivani ${ }^{26}$. También, y confirmando los hallazgos de este autor ${ }^{26}$, un tercio de mujeres de la muestra había consultado previamente con los dispositivos de salud mental.

Los hallazgos más destacados que hemos encontrado en nuestro estudio han sido los siguientes: la baja proporción de mujeres que acude a tratamiento, el inicio tardío del consumo de alcohol, la mayor rapidez en el desarrollo de la enfermedad en las mujeres, la mayor incidencia de patología depresiva y ansiosa y una morbilidad somática similar a la que presentan los hombres alcohólicos.

En relación a los objetivos que nos planteábamos en nuestro estudio parece claro que las mujeres desarrollan la enfermedad alcohólica en menos tiempo y 
sin embargo no presentan mayor morbilidad somática como muchos autores han descrito'12, 13, 21.

Un hallazgo que nos parece preocupante es la baja proporción de mujeres que acude a tratamiento. Hay tres posibles hipótesis que pueden influir en este fenómeno. En primer lugar, la percepción social negativa que siempre se ha tenido del alcoholismo en la mujer que provoca en ésta mecanismos defensivos como negación y ocultación o bien manifestándose a través de síntomas psicopatológicos (depresión y ansiedad) por lo que o no solicita ayuda o busca otros tipos de recursos, como los privados. En segundo lugar, puede que el diagnóstico de alcoholismo en la mujer se realice con menor frecuencia, debido a la menor sensibilidad de los procedimientos diagnósticos que se utilizan en la actualidad, validados probablemente en muestras masculinas. La tercera hipótesis sería la escasa detección del alcoholismo por parte de los profesionales de salud mental, cuando se presenta asociado a síntomas depresivos y ansiosos, bien por que no se sospeche o bien porque se considere secundario al trastorno depresivo o de ansiedad.

Finalmente proponemos que para detectar y diagnosticar más eficazmente un T. U. A. en la población femenina es muy importante tener en cuenta que presenta algunas características diferenciales como se ha descrito en el presente estudio: inicio tardío del consumo alcohol, ingestión de menor cantidad, curso rápido y presencia de síntomas psicopatológicos de ansiedad y de la esfera afectiva. Así mismo, la utilización de cuestionarios como CAGE, AUDIT y TWEAK, con una puntuación de corte más baja, será una ayuda de primer orden. La realización de determinadas pruebas de laboratorio como determinación de GGT, VCM, transferrina deficiente en carbohidratos (TDC) y determinación de alcohol en sangre o en el aire expirado pueden ser determinantes para el diagnóstico y deberían realizarse sistemáticamente en determinados grupos de población femenina y en determinados dispositivos sanitarios.

\section{REFERENCIAS}

1.- Grant BF, Dawson DA, Stinson FS, Chou SP, Dufour MC, Pickering RP. The 12-month prevalence and trends in DSM-IV alcohol abuse and dependence. Alcohol Research and Health 2006; 29: 79-91.

2.- Grant BF, Harford TC, Dawson DA, Chou SP, Dufour MC, Pickering RP. Prevalence of DSM-IV alcohol abuse and dependence: United States, 1992. Alcohol Health Res World 1992; 18: 243-248.

3.- Dirección General del Plan Nacional sobre Drogas. Observatorio Español sobre Drogas. Encuesta Domiciliaria sobre Abuso de drogas en España, 2003.
4.- Limusin F. Clinical and biological specificities of female alcoholism. L'Encefale 2002; 28: 503-509.

5.- Ávila JJ, Pérez A, Rodríguez M. Estudio de una muestra de pacientes alcohólicos dos años después de finalizar el tratamiento. Actas Luso-Esp Neurol Psiquiatr 1994; 22: 6-124.

6.- Beckman LJ. Women alcoholics. A review of social and psychological studies. J Stud Alcohol 1975; 36: 797-824.

7.- $\quad$ Blume S B. Women and alcohol: A review. JAMA 1986; 256: 1467-1470.

8.- Hasin DS, Grant BF, Weinflash J. Male/female differences in alcohol related problems: Alcohol rehabilitation patients. Int J Adicct 1988; 23: 437-448.

9.- Wilsnack SC, Wilsnack RW. Drinking and problem drinking in US women: Patterns and recent trends. En: Galanter M, editor. Recent Developments in Alcoholism. Volume 12. Alcoholism and Women. New York: Plenum Press; 1995. p. 29-60.

10.- Ávila JJ, Pérez A, Rodríguez M. Análisis descriptivo de una muestra de mujeres alcohólicas atendidas durante un periodo de 10 años. Adicciones 1996; 8: 429-440.

11.- Wilsnack SC. Patterns and trends in women's drinking: recent findings and some implications for prevention. En: Howard JM, Martin SE, Mail PD, Hilton ME, Taylor ED, editores. Women and Acohol: Issues for Prevention Research. National Institute on Alcohol Abuse and Alcoholism. Research Monograph No 32. Rockville MD: the Institute; 1996. p. 19-63.

12.- Blume SB. Women: clinical aspetcs. En: Lowinson JH, Ruiz P, Millman RB, Langrod JG, editores, Substance Abuse: A Comprehensive Textbook, $3^{\text {rd }}$ edition. Baltimore: Willians \& Wilkins; 1997. p. 645-654.

13.- Rubio G, Blázquez A.: Mujer y problemas por el alcohol. En: Rubio G, Santo-Domingo J, editores, Guía Práctica de Intervención en el Alcoholismo. Madrid. 2000. p. 327-336.

14.- Pfefferbaum A, Rosenbloom M, Deshmukh A, Sullivan EV. Sex differences in the effects of alcohol on brain structure. Am J Psychiatry 2001; 158: 188-197.

15.- Morales E, Camarena F, Llopis JJ. Evolución del alcoholismo en la mujer. Adiciones 1992; 4: 33-44.

16.- González F, Salvador L, Martínez JM, López A, Ruiz I, Guerra D. Estudio de fiabilidad y validez de la versión española de la entrevista clínica Addiction Severity Index (ASI). En: Iraurgi Castillo J, González F, editores, Instrumentos de Evaluación en Drogodependencias. Madrid: Aula Médica Ediciones; 2002. p. 271-307.

17.- DSM-IV. Manual diagnóstico y estadístico de los trastornos mentales. Barcelona: Masson S.A.; 1995.

18.- Bach L. Alcoholismo femenino. Adiciones 1995; 7: 3-5.

19.- Bradly KA, Boyd-Wickizer J, Powell SH, Burman ML. Alcohol screening questionnaires in women. A critical review. JAMA 1998; 280: 166-171.

20.- Wilsnack SC. Alcohol abuse and alcoholism in women. En: Pattison EM, Kaufman E, editores, Enciclopedic Handbook of Alcoholism. New York: Gradner Press; 1982. p. $718-735$. 
21.- Hill SY. Mental and physical health consequences of alcohol use in women. En: Galanter M, editor, Recent Developments in Alcoholism. Volume 12. Alcoholism and Women.. New York: Plenum Press; 1995. p. 181-197.

22.- Cole-Harding S, Wilson JR. Ethanol metabolism in men and women. J Stud Alcohol 1987; 48: 380-387.

23.- Mumenthaler MS, Taylor JL, O'Hara R, Yesavage JA. Gender differences in moderate drinking effects. Alcohol Res Health 1999; 23: 55-64.

24.- Jiang R, Hu FB, Giovannucci EL, Rimm EB, Stampfer M.J, Spiegelman D, et al. Joint Association of Alcohol and Folate Intake with Risk of Major Chronic Disease in Women. Am J Epidemiol 2003; 158: 760-771.
25.- Petrakis IL, González G, Rosenheck R, Krystal JH. Comorbidity of alcoholism and psychiatric disorders. An overview. Alcohol Res Health 2002; 26: 81-90.

26.- Shivani R, Goldsmith J, Anthenelli RM. Alcoholism and psychiatric disorders: Diagnostic challenges. Alcohol Res Health 2002; 26: 90-98.

27.- Kessler RC, Crum RM, Warner LA. Life-time co-occurence of DSM-III-R alcohol abuse and dependence with other psychiatric disorders in the National Comorbidity Survey. Arch Gen Psychiatry 1997; 54: 313-321. 